\title{
Synchronous tumors of the pancreas and the gallbladder: a case report with targeted NGS evaluation
}

\author{
Aurelie Sivade ${ }^{1}$, Christine Sempoux ${ }^{2}$, Ioannis Voutsadakis ${ }^{3}$, Christophe Brunel ${ }^{2}$, Nermin Halkic ${ }^{4}$, \\ Sebastian Godat ${ }^{5}$, Rafael Duran ${ }^{6}$, Antonia Digklia ${ }^{1}$ \\ ${ }^{1}$ Department of Oncology, Lausanne University Hospital, University of Lausanne, Lausanne, Switzerland; ${ }^{2}$ Department of Pathology, Lausanne \\ University Hospital, University of Lausanne, Lausanne, Switzerland; '3 Section of Internal Medicine, Division of Clinical Sciences, Northern Ontario \\ School of Medicine, Sudbury, ON, Canada; ${ }^{4}$ Department of Visceral Surgery, Lausanne University Hospital, University of Lausanne, Lausanne, \\ Switzerland; '5epartment of Gastroenterology, Section of Internal Medicine, Lausanne University Hospital, University of Lausanne, Lausanne, \\ Switzerland; ${ }^{6}$ Department of Diagnostic and Interventional Radiology, Lausanne University Hospital, University of Lausanne, Lausanne, Switzerland \\ Correspondence to: Antonia Digklia, MD, MSc. Rue du Bugnon 46, 1011, Lausanne, Switzerland. Email: antonia.digklia@chuv.ch.
}

\begin{abstract}
Synchronous tumors of the pancreas and gallbladder are rare and often attributed to an abnormal pancreato-biliary junction, which results in a persistent reflux of pancreatic secretions leading to chronic biliary inflammation. We present the case of a 73-year-old woman with synchronous lesions of the pancreas and gallbladder initially considered as two primary localized cancers and treated with curative intent. At relapse, targeted next generation sequencing (NGS), performed in search of potential therapeutic targets, uncovered the fact that the two lesions appeared to be clonally related. This case illustrates the problem of synchronous lesions of the pancreas and gallbladder. New pathologic assessments with comparative molecular analysis of mutational profiles may be helpful in this context.
\end{abstract}

Keywords: Next generation sequencing (NGS); gallbladder; cancer; HER2

Submitted Mar 26, 2019. Accepted for publication Aug 08, 2019.

doi: 10.21037/atm.2019.10.107

View this article at: http://dx.doi.org/10.21037/atm.2019.10.107

\section{Introduction}

Patients with synchronous tumors are a well-recognized and frequently encountered problem in clinical oncology. Currently there is no consensus on the definition of synchronous cancer. Among the proposed definitions are: "two or more histologically distinct simultaneously detected malignancies" or "two or more histologically distinct malignancies arising in the same site, following each other in sequence by less than 2 months" (1). In such cases it is important to establish whether the tumors have arisen independently of each other or whether one lesion is the metastasis of the other, since the prognosis and clinical management may differ in each situation.

Independent primary pancreato-biliary tumors are rare and most are thought to be associated with pancreatobiliary reflux (2). Only seven cases have been reported with simultaneous cancers of gallbladder and pancreas
(Table 1). Pancreatic metastases are very uncommon and are only found in a minority $(3-12 \%)$ of patients with widespread metastatic disease at autopsy. They account for only $2-5 \%$ of all pancreatic malignancies and the most common primaries encountered include kidney, melanoma and breast cancer (10).

Gallbladder and extra-hepatic biliary cancer is a relatively rare disease with an estimated 12,360 new cases in the United States in 2019. Its incidence increases with age and is slightly more common in women (11). The liver and the peritoneal cavity are common sites of metastases in gallbladder cancer (GBC). The lungs and the bones are less commonly involved. Unusual sites of metastases such as muscle, brain, kidney and uterus have also been reported in GBC (12).

Below we present a case of synchronous lesions of the pancreas and gallbladder managed as two separate primary tumors. After recurrence with liver metastasis, the mutational profiles of these two tumor tissues were 
Table 1 Cases reports of simultaneous GBC and PC

\begin{tabular}{|c|c|c|c|c|c|}
\hline Reference & Gender/age/race & Diagnosis & $\begin{array}{l}\text { Location } \\
\text { pancreas }\end{array}$ & Treatments & Survival \\
\hline Ueda et al. 1992 (3) & Male 58 Thai & $\begin{array}{l}\text { Synchronous } \\
\text { (GBC, PC, BDC) }\end{array}$ & Diffuse & $\begin{array}{l}\text { Total pancreatectomy and } \\
\text { extended cholecystectomy }\end{array}$ & No information \\
\hline Minami et al. 2008 (4) & Female 50 Japanese & $\begin{array}{l}\text { Metachronous } \\
\text { (GBC then PC) }\end{array}$ & Tail & $\begin{array}{l}\text { Cholecystectomy then } \\
\text { spleno-pancreatectomy }+ \\
\text { partial gastrectomy and left } \\
\text { adrenalectomy }\end{array}$ & $\begin{array}{l}\text { Still alive after } \\
6.5 \text { years }\end{array}$ \\
\hline Lahmar et al. 2010 (5) & Female 68 Tunisian & $\begin{array}{l}\text { Metachronous } \\
\text { (GBC then PC) }\end{array}$ & Head & $\begin{array}{l}\text { Extended cholecystectomy } \\
\text { with adjuvant chemotherapy } \\
\text { (cisplatin/5 FU) then } \\
\text { pancreatoduodenectomy }\end{array}$ & $\begin{array}{l}\text { Still alive after } \\
1 \text { year }\end{array}$ \\
\hline Sato et al. 2003 (6) & Man 74 Japanese & $\begin{array}{l}\text { Synchronous } \\
\text { (GBC, CBDC, PC) }\end{array}$ & $?$ & Pancreatoduodenectomy & $\begin{array}{l}\text { Died of recurrence } \\
\text { after } 1 \text { year }\end{array}$ \\
\hline Agarwal et al. 2013 (8) & Female 35 Indian & $\begin{array}{l}\text { Synchronous } \\
\text { (GBC, PC) }\end{array}$ & Head & Cholecystectomy & $\begin{array}{l}\text { Died } 25 \text { days after } \\
\text { surgery }\end{array}$ \\
\hline Mori et al. 2017 (9) & Female 72 Japanese & $\begin{array}{l}\text { Synchronous } \\
\text { (GBC, PC) }\end{array}$ & Head & $\begin{array}{l}\text { Pancreatoduodenectomy }+ \\
\text { cholecystectomy }\end{array}$ & $\begin{array}{l}\text { Died } 8 \text { months after } \\
\text { surgery }\end{array}$ \\
\hline
\end{tabular}

GBC, gallbladder cancer; PC, pancreatic cancer.

compared and suggested that the pancreatic mass was possibly a metastasis of the GBC.

\section{Case presentation}

A 73-year-old Caucasian woman with a history of hypercholesterolemia presented with acute painless jaundice. Personal and family history was negative for neoplastic diseases. Laboratory results upon admission revealed elevated transaminases (ASAT 393 U/L, ALAT $939 \mathrm{U} / \mathrm{L}$ ), and icteric cholestasis (GGT $633 \mathrm{U} / \mathrm{L}$, bilirubin $64.9 \mathrm{umol} / \mathrm{L})$. The complete blood count, creatinine and electrolytes were normal. A Computed tomography (CT) of the abdomen with contrast was performed showing dilated bile ducts due to the presence of a mass in the head of the pancreas. Moreover, signs of acute cholecystitis with thickened gallbladder wall and multiple gallstones were described but no distant metastatic lesions (Figure 1). Endoscopic retrograde cholangiopancreatography (ERCP) showed a stenosis (length of $13 \mathrm{~mm}$ ) at the lower third of major biliary tract and a stent was placed.
Fine needle aspiration cytology (FNAC) of the pancreatic mass confirmed the existence of a ductal adenocarcinoma (PDAC). PET/CT scan was performed revealing a moderate degree of 18-fluorodeoxyglucose (FDG) uptake in the pancreas head (SUVbw max.: 10.9 and at the wall of the gallbladder (SUVbw max.: 11.2), interpreted as of possible infectious etiology with no other suspicious foci (Figure 2). The Ca 19-9 level was normal.

Therefore, clinical staging was stage III (T4N0M0) and the patient underwent cephalic pancreato-duodenectomy with resection of the gallbladder and bilio-enteric anastomosis at the level of convergence (Whipple). No frozen sections were performed intraoperatively and the gallbladder wall thickening was attributed to cholecystitis due to obstruction from the pancreatic head carcinoma and possibly from cholelithiasis. Surprisingly, pathological examination revealed the presence of two different tumors: a moderately differentiated ductal adenocarcinoma of the pancreas [pT2 pN1 (3/14)] with lymphovascular invasion and perineural infiltration as well as positive margins at the mesenteric vein, associated with pancreatic intraepithelial 

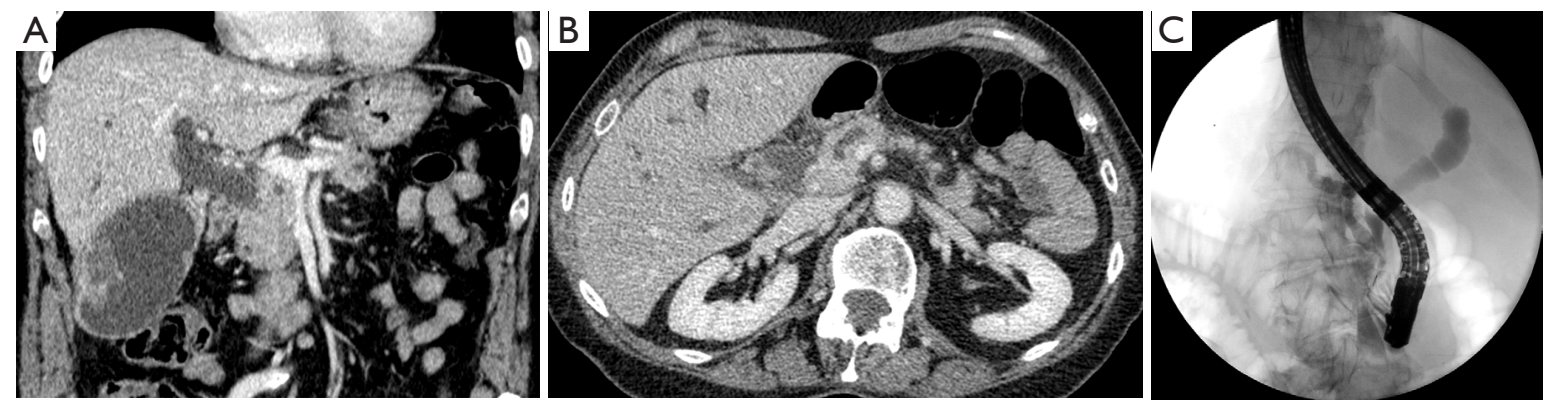

Figure 1 CT images. (A) Coronal cut: pancreatic mass with double duct sign. (B) Axial cut: Wirsung dilation due to a pancreatic mass. (C) ERCP demonstrating a classic "double duct" sign. CT, computed tomography; ERCP, endoscopic retrograde cholangiopancreatography.
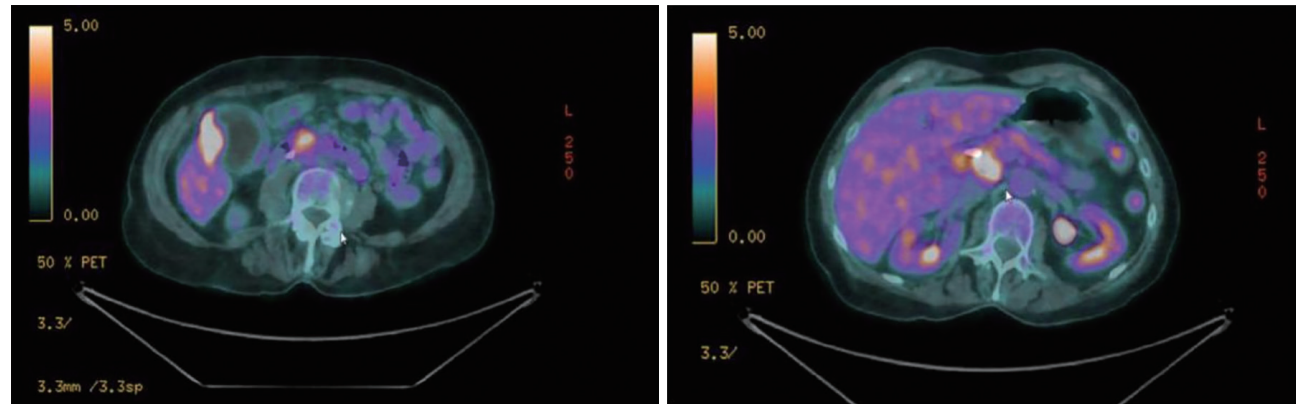

Figure 2 18-FDG PET/ CT scan at the level of GB and pancreatic head before surgery.

neoplasia of grade 1 in intralobular pancreatic ducts and a poorly differentiated adenocarcinoma of the gallbladder classified pT3 pN1 (3/5) V1 L1 Pn1 R0, associated with high grade biliary intraepithelial neoplasia (BilIN3). Adjuvant chemotherapy with Gemcitabine for six months was given with excellent clinical tolerance.

Unfortunately, six months after the end of the adjuvant therapy, a follow up CT showed a single liver metastasis in segment $\mathrm{V}$, confirmed by MRI and by biopsy. The CA 19-9 serum level was normal. After multidisciplinary discussion, a first line palliative chemotherapy with the FOLFOX regimen was started. Five months later the patient had disease progression with appearance of retroperitoneal lymph node and lung metastases. In this context, and in the absence of standard second line or clinical trial options, we performed a next generation sequencing (NGS) analysis (customized NGS panel covering 218 hotspot regions of 52 genes from panel Ion Torrent) on the tumors of the gallbladder and the pancreas, in order to search for potential therapeutic targets (Figure 3). We also assessed HER2 status by immunohistochemistry (IHC) and FISH in the three disease localizations for which tissue was available (gallbladder, pancreas and liver).
NGS analyses revealed a common TP53 gene mutation (c.742C>T/p.R248W in exon 7, OncoKB: Loss of function, likely oncogenic) in the gallbladder and pancreatic tumors, with an additional PTEN gene mutation restricted to the gallbladder lesion (c.395G>A/p.G132D in exon 5, OncoKB: no available functional data but statistically significant hotspot and is predicted to be oncogenic). Of note, no KRAS mutation was identified (Table 2). HER2 status was heterogeneously positive in the gallbladder lesion, with an amplified component and another region characterized by an equivocal result both by IHC and by FISH. In the pancreatic tumor HER2 status was equivocal (by IHC and FISH), while the hepatic lesion showed a low-grade amplification.

Altogether, these data suggested the existence of a clonal relationship between the three lesions. Although the precise chronology of events could not be established, we favoured the hypothesis that the starting point was a gallbladder adenocarcinoma (developed in association with high-grade biliary intraepithelial neoplasia), characterized by a genetic heterogeneity and a divergent evolution of two different subclones: one subclone metastasized to the pancreas, the other subclone acquired a HER2 gene amplification (found 


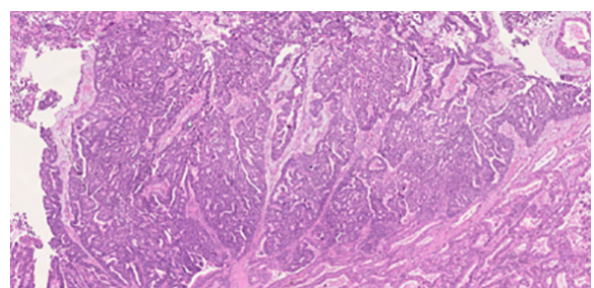

Gallbladder

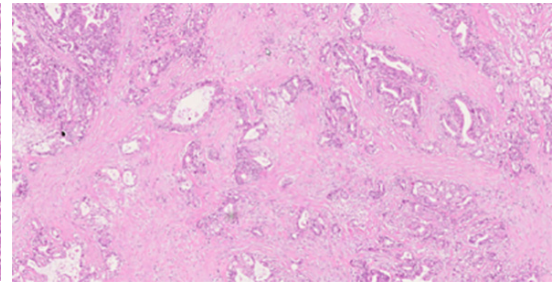

Pancreas

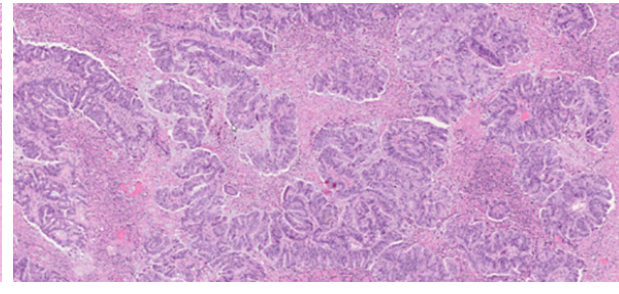

Liver metastasis

Figure 3 Pathology images. Hematoxylin and eosin stained sections, 100× original magnification.

Table 2 NGS panel findings

\begin{tabular}{lllll}
\hline Gene & Gallbladder lesion & Pancreatic lesion & Brain metastasis & Liver metastasis \\
\hline TP53 & c.742C $>\mathrm{T}$ & c.742C $>\mathrm{T}$ & c.742C $>\mathrm{T}$ & Not done \\
KRAS & No mutation & No mutation & No mutation & Aone \\
ERBB2 & $\begin{array}{l}\text { Amplification }>19 \text { copies/cell } \\
\text { (equivocal in some areas) }\end{array}$ & Equivocal & Amplification $>55$ copies/cell & Not done \\
PTEN & c.395G $>\mathrm{A}$ & No mutation & No mutation & Not done \\
Other & & & EGFR, GNAS amplification CDH1 deletion & N \\
\hline
\end{tabular}

NGS, next generation sequencing.

in a component of the gallbladder tumor and to a lesser extent in the liver metastasis).

Based on these results, we added trastuzumab (antiHER2 antibody) to our backbone second line palliative chemotherapy (FOLFIRI), with stable disease for 5 months. At the disease progression, we treated the patient with the combination of trastuzumab/lapatinib (tyrosine kinase inhibitor of EGFR and HER2), as well as with paclitaxel/ trastuzumab. Unfortunately, the patient presented with brain symptomatic metastasis treated by surgery followed by rapid deterioration of her general condition and died four weeks later. The NGS analysis of the brain metastasis revealed the common TP53 gene mutation present in the other locations as well as an EGFR mutation and a strong amplification of the HER2.

\section{Discussion}

Only a few cases have been reported in the literature of concomitant or metachronous pancreatic carcinoma and GBC (Table 1). Interestingly all cases but one were from Asia and all cases but one for which information is available involved the head of the pancreas. In our case the clinical challenge was to make the distinction between pancreaticobiliary primary tumor with related metastatic lesions or two independent primary pancreatico-biliary tumors since both of them have similar histologic appearance and immunophenotypes, mainly regarding the expression of the same cytokeratin panel. An additional challenge in our case stemmed from the presence of the pancreatic mass, biliary dilatation and gallstones that diverted suspicion from a gallbladder carcinoma till the pathology of the cholecystectomy was available. GBC has nonspecific symptoms including pain, anorexia, nausea, or vomiting often leading to a diagnosis only at the time of surgical exploration for abdominal symptoms attributable to acute or chronic cholecystitis. In fact an incidental finding of GBD in cholecystectomies has been reported in $0.2 \%$ to $2.5 \%$ in various series. Ultrasonography (US) for diagnosis of GBC has limited sensitivity and additional imaging [CT or magnetic resonance imaging (MRI)] is needed for patients who have concerning findings on US (mass protruding into the lumen, loss of interface between gallbladder and liver, gallbladder polyps $\geq 10 \mathrm{~mm}$, direct liver infiltration, thickened gallbladder wall that is not explained by cholecystitis).

Recent technological advances such as NGS may be helpful for the determination of whether there is a clonal relationship between different tumor tissues from the same patient. 
GBC have a spectrum of mutations including more commonly TP53, KRAS, PIK3CA (13), but these overlap with pancreatic carcinomas. However frequencies of these mutations defer.

TP53 is a tumor suppressor gene and its mutations are found in a majority of human malignancies. TP53 mutations were seen in $73.9 \%$ of pancreatic cancer patients and in $47 \%$ of GBC $(14,15)$.

$K R A S$ mutations in biliary tract cancer have been found commonly in the setting of anomalous junction of pancreatic and biliary ducts. KRAS mutations have been implicated in $>95 \%$ of PDAC and are considered as an early or initiating event for PDAC carcinogenesis (16). Mutations most commonly occur at codons 12 (54-74\%), 13 (3-5\%), and $61(3-5 \%)$. On the other hand, a low incidence of $K R A S$ mutations has been described in most studies of GBC in western countries (0-10\%), although the data from Japanese studies are more variable (incidence $0-59 \%)(15,17)$. In this context, the absence of KRAS mutation in the analysis of the pancreatic lesion, although it is not conclusive, points against a pancreatic primary.

PTEN is a major tumor suppressor and inhibitor of the PI3K-Akt kinase pathway but mutations are uncommon in pancreatic ductal adenocarcinoma where it regulates an NF-kappaB-centered cytokine network (18). PTEN mutations are also rare in GBC. In the recent analysis of Ali and al of 141 cases of GBC, it has been showed that PTEN mutations occur in $4.8 \%$ of patients and all of them where observed in exon 9. The presence of mutation exon 5 is common in other cancers and a possible allelic variation of PTEN gene in different ethnic groups has been observed (19).

HER2 amplification occurred in as many as $14 \%$ of the advanced GBC cases. Interestingly, it had been shown that recurrent mutations in the ErbB pathway are linked with worse outcome. Clinical data suggest that a HER2-targeting strategy may be beneficial in this group of patients $(20,21)$.

Given the above observations, and given the genetic heterogeneity and divergent evolution derived from the NGS genomic studies, we favour the hypothesis that the starting point was the gallbladder. This primary tumor acquired amplifications of HER2 in some areas which metastasized to the liver and brain while other clones without HER2 amplification travelled across the biliary tree and developed invasive metastasis in the pancreas.

The prognosis of metastatic gallbladder is poor, with a median survival of less than 12 months (22). Few case reports have reported interesting outcomes after surgery for selected oligometastatic patients (3-9). Surgical resection can be performed safely in selected patients with isolated metastases to the para-aortic lymph node or isolated liver metastasis, achieving long-term survival as well as good palliation (23). In our case, the patient benefited from an HER2-targeting strategy with a long survival of 3 years.

In summary, the pancreas represents an uncommon but clinically significant site of metastasis in gallbladder adenocarcinoma when simultaneous lesions are found. NGS can provide valuable information in the workup of these cases, when available. However, given the high cost of NGS at this time, a cost-benefit analysis should be considered before routine use. In our case, use of a targeted NGS panel led to use of targeted treatments and resulting in meaningful prolongation of patient's survival. In addition, the case illustrates the usefulness of PET scanning in evaluation of hepato-biliary tumors. However, one has to be careful before ascribing increased up-take to infection or inflammation. Finally, in cases where there is a solitary metastasis of the gallbladder to the pancreas, as in our case, and if radical simultaneous resection is feasible, it should be considered after discussion in a multidisciplinary board.

\section{Acknowledgments}

None.

\section{Footnote}

Conflicts of Interest: The authors have no conflicts of interest to declare.

Ethical Statement: The authors are accountable for all aspects of the work in ensuring that questions related to the accuracy or integrity of any part of the work are appropriately investigated and resolved. Written informed consent was obtained from the patients for publication of this Case reports and any accompanying images.

\section{References}

1. Luciani A, Balducci L. Multiple primary malignancies. Semin Oncol 2004;31:264-73.

2. Benjamin IS. Biliary cystic disease: the risk of cancer. J Hepatobiliary Pancreat Surg 2003;10:335-9.

3. Ueda N, Nagakawa T, Ohta $T$ et al. Synchronous cancer of the biliary tract and pancreas associated with anomalous 
arrangement of the pancreaticobiliary ductal system. J Clin Gastroenterol 1992;15:136-41.

4. Minami Y, Hasuike Y, Takeda Y. et al. Metachronous double cancer of the gallbladder and pancreas associated with pancreaticobiliary maljunction. J Hepatobiliary Pancreat Surg 2008;15:330-3.

5. Lahmar A, Abid SB, Arfa MN, et al. Metachronous cancer of gallbladder and pancreas with pancreatobiliary maljunction. World J Gastrointest Surg 2010;2:143-6.

6. Sato K, Maekawa T, Yabuki K et al. A case of triple synchronous cancers occurring in the gallbladder, common bile duct, and pancreas. J Gastroenterol 2003;38:97-100.

7. Rungsakulkij N, Boonsakan P. Synchronous gallbladder and pancreatic cancer associated with pancreaticobiliary maljunction. World J Gastroenterol 2014;20:14500-04.

8. Agarwal N, Kumar S, Sharma S. Synchronous adenocarcinoma of the gall bladder and pancreas in a young woman. Trop Gastroenterol 2013;34:50-2.

9. Mori $\mathrm{H}$, Iida $\mathrm{H}$, Maehira $\mathrm{H}$, et al Synchronous primary gallbladder and pancreatic cancer associated with congenital biliary dilatation and pancreaticobiliary maljunction. Surg Case Rep 2017;3:113.

10. Triantopoulou C, Kolliakou E, Karoumpalis I, et al. Metastatic disease to the pancreas: an imaging challenge. Insights Imaging 2012;3:165-72.

11. Siegel RL, Miller KD, Jemal A. Cancer statistics. CA Cancer J Clin 2019:69:7-34

12. Sons HU, Borchard F, Joel BS. Carcinoma of the gallbladder: autopsy findings in 287 cases and review of the literature. J Surg Oncol 1985;28:199-206.

13. Li M, Zhang Z, Li X. et al. Whole-exome and targeted gene sequencing of gallbladder carcinoma identifies recurrent mutations in the ErbB pathway. Nat Genet 2014;46:872.

Cite this article as: Sivade A, Sempoux C, Voutsadakis I, Brunel C, Halkic N, Godat S, Duran R, Digklia A. Synchronous tumors of the pancreas and the gallbladder: a case report with targeted NGS evaluation. Ann Transl Med 2019;7(22):696. doi: 10.21037/atm.2019.10.107
14. Cicenas J, Kvederaviciute K, Meskinyte I, et al. KRAS, TP53, CDKN2A, SMAD4, BRCA1, and BRCA2 mutations in pancreatic cancer. Cancers 2017;9:42.

15. Iacobuzio-Donahue CA, Velculescu V, Wolfgang CL, et al. Genetic Basis of Pancreas Cancer Development and Progression: Insights from Whole-Exome and WholeGenome Sequencing. Clin Cancer Res 2012;18:4257-65.

16. Ajiki T, Fujimori T, Onoyama H, et al. K-ras gene mutation in gall bladder carcinomas and dysplasia. Gut 1996;38:426-29.

17. Tada M, Yokosuka O, Omata M, et al. Analysis of ras gene mutations in biliary and pancreatic tumors by polymerase chain reaction and direct sequencing. Cancer 1990;66:930-5.

18. Ying H, Elpek KG, Vinjamoori A, et al. PTEN is a major tumor suppressor in pancreatic ductal adenocarcinoma and regulates an NF- $\mathrm{kB}$-cytokine network. Cancer Discov 2011;1:158-69.

19. Ali A, Mishra PK, Sharma S, et al. Effects of PTEN gene alteration in patients with gallbladder cancer. Cancer Genet 2015;208:587-94.

20. Roa I, de Toro G, Schalper K, et al. Overexpression of the HER2/neu gene: a new therapeutic possibility for patients with advanced gallbladder cancer. Gastrointest Cancer Res 2014;7:42.

21. Nam AR, Kim JW, Cha Y, et al. Therapeutic implication of HER2 in advanced biliary tract cancer. Oncotarget 2016;7:58007.

22. Kanthan R, Senger JL, AhmedS, et al. Gallbladder cancer in the 21st century. J Oncol 2015;2015:967472.

23. Nishio H, Nagino M, Ebata T, et al. Aggressive surgery for stage IV gallbladder carcinoma; what are the contraindications? J Hepatobiliary Pancreat Surg 2007;14:351-7. 